\title{
Associação entre Ângulo de Fase, PRISM I e Gravidade da Sepse*
}

\section{Association between Phase Angle, PRISM I and Sepsis Severity}

\author{
Zina Maria Almeida de Azevedo', Dilma Ribeiro Silva², Maria Virgínia P. Dutra ${ }^{3}$, \\ Maria Ignêz C. Gaspar Elsas ${ }^{4}$, Maria Cristina G. Barbosa-Silva ${ }^{5}$, Vânia Matos Fonseca ${ }^{6}$
}

\section{RESUMO}

JUSTIFICATIVA E OBJETIVOS: Ângulo de fase (AF) é a diferença entre a voltagem e a corrente e pode ser usado como indicador de massa celular corporal. Estudos clínicos mostram que baixos AF estão associados com morbidade e mortalidade em pacientes críticos. $O$ objetivo deste estudo foi conhecer a relação entre AF e o escore pediátrico de risco de mortalidade (PRISM I) em pacientes pediátricos sépticos críticos, associando esse indicador $\mathrm{c}$ om a gravidade da sepse.

MÉTODO: Estudo transversal realizado na Unidade de

1. Médica, Chefe da Unidade de Pacientes Graves do Instituto Fernandes Figueira - FIOCRUZ, Doutora em Ciências, Professora Adjunta Doutor I da Universidade UNIGRANRIO.

2. Nutricionista

3. Professora e Pesquisadora da Pós-Graduação em Saúde da MuIher e da Criança - FIOCRUZ. Doutora em Engenharia Biomédica.

4. Pesquisadora Titular da FIOCRUZ, Bolsista CNPQ em Produtividade. Doutora em Patologia.

5. Professora Adjunta da Escola de Medicina da Universidade Católica de Pelotas. Doutora em Epidemiologia.

6. Professora e Pesquisadora da Pós-Graduação em Saúde da MuIher e da Criança - FIOCRUZ. Doutora em Epidemiologia.

*Recebido do Instituto Fernandes Figueira, FIOCRUZ (Departamento de Pediatria, Núcleo de Epidemiologia e Laboratório de Fisiopatologia Humana), Rio de Janeiro, RJ

- Programa de Pós-Graduação em Epidemiologia, Universidade Federal de Pelotas, Rio Grande do Sul, RS.

- Programa de Desenvolvimento Tecnológico em Saúde (PDTSP)Fundação Oswaldo Cruz e Instituto Fernandes Figueira.

Apresentado em 07 de fevereiro de 2007

Aceito para publicação em 20 de junho de 2007

Endereço para correspondência:

Dra. Zina Maria Almeida de Azevedo

Av. Rui Barbosa 716 - Flamengo

22250-020, Rio de Janeiro, RJ

Fone: (21) 2554-1873, Fax: (21) 2553-8094.

E-mail: zina@iff.fiocruz.br, zina maria@uol.com.br

(C)Associação de Medicina Intensiva Brasileira, 2007
Terapia Intensiva (UTI) Pediátrica do Instituto Fernandes Figueira. Os pacientes foram caracterizados de acordo com faixa etária, sexo, gravidade da sepse, etiologia da insuficiência respiratória, escore de PRISM I, grau de disfunção de múltiplos órgãos e sistemas (DMOS). A análise de bioimpedância elétrica (BIA) foi realizada em todos os pacientes e, através da razão dos valores de reactância $(X c)$ e resistência $(R)$, foi calculado o $A F(A F=$ arco-tangente da reactância/resistência x $180^{\circ} / \mathrm{Pi}$ ).

RESULTADOS: Foram avaliados 75 pacientes, sendo $68(90,7 \%)$ com sepse. A incidência de choque séptico foi $39,7 \%$, sepse grave $42,6 \%$ e sepse $17,6 \%$. Não houve diferença estatística significativa entre as médias de ângulo de fase e as categorias de PRISM I, porém observou-se uma relação inversa entre os valores de AF e as categorias de PRISM I, DMOS e tempo de internação. Os valores mais baixos de $\operatorname{AF}\left(1,5^{\circ}-2,2^{\circ}\right)$ foram observados no maior escore de PRISM I (> $30 \%$ ).

CONCLUSÕES: Os pacientes pediátricos críticos apresentaram baixos valores de angulo de fase, portanto deve ter a sua importância prognóstica estudada.

Unitermos: ângulo de fase, escore prognóstico, impedância elétrica, PRISM I, UTI pediátrica.

\section{SUMMARY}

BACKGROUND AND OBJECTIVES: Phase angle (PA) is the difference between voltage and current and can be used as an indicator of body cell mass. Clinical studies show that low phase angle is associated with morbidity and mortality of critical patients. The purpose of this study was to know the relation between phase angle and the Pediatric Risk of Mortality I (PRISM I) score, associating this score with the severity of sepsis.

METHODS: A transversal study was performed at the Pediatric Intensive Care Unit (PICU) in Instituto Fernandes Figueira. The patients were classified according to age, gender, sepsis severity, cause of respiratory failure, PRISM I score, multiple organ 
dysfunction syndromes (MODS). Electrical bioimpedance analysis (BIA) was performed in all patients. Phase angle was calculated directly from reactance (Xc) and resistance (R). AF = arc-tangent reactance/ resistance $\mathrm{x} 180^{\circ} / \mathrm{Pi}$.

RESULTS: 75 patients (68 septic) were evaluated. The incidence of septic shock was $39.7 \%$, severe sepsis $42.6 \%$ and sepsis $17.6 \%$. There was no significative statistical difference between the mean values of BIA and the categories of PRISM I, MODS, or the length of stay the PICU. The PA's lowest values $\left(1.5^{\circ}-2.2^{\circ}\right)$ were associated to the greatest PRISM's scores (> 30\%).

CONCLUSIONS: Pediatric critical patients show low phase angle values, which might have prognostic implication.

Key Words: electrical bioimpedance analysis, phase angle; PRISM I score; PICU.

\section{INTRODUÇÃO}

Os modelos para predizer risco de morte em grupos de pacientes internados em unidades de terapia intensiva (UTI), ajustados para as diferenças na gravidade das doenças e diagnósticos, podem ser usados para comparar os padrões de cuidados entre unidades, ou dentro de uma mesma unidade ao longo do tempo ${ }^{1}$. Os escores de risco de mortalidade (através de escala numérica) comparam a probabilidade de mortalidade estimada com a mortalidade realmente observada. Por compararem a mortalidade ajustada à gravidade da doença, servem para a classificação desses pacientes ${ }^{2}$.

O escore Pediatric Risk of Mortality (PRISM I), usado em UTI pediátrica na última década, é apontado por muitos estudos como válido e confiável ${ }^{3}$. Esse escore foi desenvolvido a partir da identificação de variáveis relevantes para o risco de mortalidade, ponderadas após análise multivariada de regressão logística ${ }^{4}$. Uma versão revisada do PRISM I, o PRISM III, está disponível desde 1996 e, segundo seus autores, fornece maior capacidade preditiva ${ }^{3,5}$.

A bioimpedância (BIA) é um método simples, rápido e não-invasivo para avaliar composição corporal. Estima volumes hídricos corporais, baseados na condutividade de uma corrente elétrica de $50 \mathrm{kHz}$ e $800 \mathrm{~mA}$ através do corpo, onde a massa magra e a água corporal total são condutores de corrente elétrica e a gordura corporal oferece resistência à sua passagem. A avaliação da composição corporal, usando a BIA, é baseada em equações de regressão desenvolvidas a partir de comparações com métodos de referência (medida de absorção de dupla energia de raios X (DEXA), hidrodensitometria e radioisótopos que usam os parâmetros resistência $(R)$, reactância $(X c)$ e outras variáveis como peso, idade, sexo e altura para estimar a água corporal, a massa magra, a massa celular corporal e a gordura corporal $^{6,7}$. No entanto, as estimativas realizadas através da BIA baseiam-se em dois pressupostos: que a hidratação dos tecidos é igual em todos os indivíduos, e que o corpo humano se comporta como um cilindro que homogeneamente conduz a corrente elétrica. Assim, a BIA não é um bom método para avaliação da composição corporal nas situações onde esses dois princípios não sejam válidos; tais como obesidade, pacientes críticos e distúrbios de hidratação, como ascite e edema ${ }^{8-11}$.

Ângulo de fase (AF) é a diferença entre a voltagem e a corrente e pode ser usado como indicador de massa celular corporal. Ele pode ser obtido diretamente através da razão dos valores de Xc e R. Estudos clínicos mostram que baixos ângulos de fase e baixos níveis de massa celular corporal estão associados com morbidade e mortalidade em pacientes críticos ${ }^{8}$. Embora o significado biológico do ângulo de fase não seja completamente compreendido, ele reflete não somente a massa celular corporal, mas também é um dos melhores indicadores da função da membrana celular, relacionado à razão entre água extracelular e água intracelular ${ }^{11}$. Ângulo de fase pode ser usado como indicador nutricional em adultos e crianças ${ }^{12}$. Uma associação positiva entre o ângulo de fase e vários tipos de doenças graves sugere que este pode ser um importante instrumento para estimar resultados clínicos ou monitorar pacientes críticos ${ }^{8,12-16}$.

O objetivo deste estudo foi conhecer a relação entre ângulo de fase e escore PRISM I em pacientes pediátricos sépticos críticos, associando este indicador com a gravidade da sepse.

\section{MÉTODO}

Estudo transversal realizado em pacientes admitidos no período de abril de 2004 a dezembro de 2005, na UTI pediátrica - Unidade de Pacientes Graves (UPG) - do Departamento de Pediatria do Instituto Fernandes Figueira (IFF), Fundação Oswaldo Cruz (FIOCRUZ). A população foi constituída por pacientes críticos admitidos pela gravidade de seus quadros clínicos, devidos principalmente à insuficiência respiratória, quadros in- 
fecciosos e instabilidade nos casos de pós-operatório de cirurgia pediátrica e neurocirurgia. $\mathrm{O}$ protocolo foi aprovado pelo Comitê de Ética da Instituição e o consentimento informado foi obtido de todos os responsáveis. A população foi caracterizada sócio-demograficamente e pelas informações clínicas, tais como doença de base preexistente; gravidade do quadro infeccioso (sepse, sepse grave, choque séptico, segundo o International Pediatric Sepsis Consensus Conference ${ }^{17}$ ); etiologia da insuficiência respiratória: lesão pulmonar aguda, síndrome do desconforto respiratório agudo (SDRA), suporte ${ }^{18,19}$; escore prognóstico padrão para avaliação da gravidade de doença em pacientes pediátricos (PRISM I) 2,20; grau de disfunção de múltiplos órgãos e sistemas (DMOS) ${ }^{17}$. As cinco categorias de PRISM I foram definidas de acordo com a probabilidade de óbito conforme descrito no estudo de Gemke e van Vught ${ }^{20}$.

Todos os pacientes foram tratados de acordo com a rotina assistencial da UPG, a avaliação nutricional e da BIA passaram a fazer parte essa rotina, sendo realizadas nas primeiras $48 \mathrm{~h}$ de admissão. O exame de BIA foi realizado com o aparelho BIA 101 Quantum II (RJL Inc., Estados Unidos), através da passagem de uma corrente alternada de baixa freqüência e alta voltagem (800 mA e $50 \mathrm{kHz})$, indolor e totalmente segura, aplicada na mão e no pé. A impedância corporal total foi obtida através da mensuração da Xc e da $R$ em Ohms $(\Omega)$. O ângulo de fase $(\phi)$ foi obtido a partir da relação arco-tangente da reactância/resistência x $180^{\circ} / \mathrm{Pi}$ cuja fórmula foi: $\phi=$ arco tangente (Xc/R). Para transformar o resultado de radiano para graus $\left({ }^{\circ}\right)$, multiplicou-se o resultado obtido por $180^{\circ} / \mathrm{Pi}$ ou aproximadamente $57,296^{10,21}$. De acordo com a descrição da técnica, eletrodos adesivos foram colocados em locais previamente padronizados na mão e no pé: um eletrodo interno (sensitive) foi colocado na superfície dorsal do punho direito entre os ossos ulnar e rádio e um eletrodo externo (injecting) colocado no terceiro metacarpo; um eletrodo interno da perna colocado na superfície anterior do tornozelo direito entre as porções proeminentes dos ossos e um quarto eletrodo, colocado na superfície dorsal do terceiro metacarpo $8,10,12,15$. Cuidados especiais foram observados em relação à colocação dos eletrodos, decúbito do paciente e a não interferência no campo elétrico.

Todos os dados clínicos e de BIA foram coletados em formulário padronizado e colocados em banco de dados do programa EPIINFO 2000. Os testes $t$ de Student, a Análise de Variância, o Qui-quadrado e testes estatísticos não paramétricos foram utilizados para descrever e analisar as associações entre os dados sumarizados através de médias e/ou medianas e de proporções. Verificou -se também a relação linear através da regressão entre valores de variáveis contínuas como o AF, PRISM I e dias de internação.

\section{RESULTADOS}

\section{Caracterização Clínica}

Foram avaliados 75 pacientes e suas características estão apresentadas na tabela 1. A ventilação mecânica foi instituída em todos os pacientes com sepse. Ao avaliar a média de tempo de internação nos grupos de sepse, observou-se diferença estatisticamente significativa entre pacientes com choque séptico, sepse grave e sepse (Tabela 2), sendo que o tempo médio de internação dos pacientes com choque séptico foi duas vezes mais prolongado que os pacientes com sepse.

Tabela 1 - Características dos Pacientes

\begin{tabular}{lcc}
\hline Variáveis & $\mathrm{N}$ & $\%$ \\
\hline $\begin{array}{l}\text { Sexo } \\
\quad \text { Masculino/Feminino }\end{array}$ & $37 / 38$ & $49,3 / 50,7$ \\
$\begin{array}{l}\text { Doenças de base } \\
\quad \text { Respiratória }\end{array}$ & 27 & 36 \\
$\quad$ Neurológica & 19 & 25,3 \\
$\quad$ Digestiva & 17 & 22,7 \\
$\quad$ Outras & 12 & 16 \\
Sepse & 68 & 90,7 \\
Gravidade da sepse & & \\
$\quad$ Sepse & 12 & 17,6 \\
$\quad$ Choque séptico & 27 & 39,7 \\
$\quad$ Sepse grave & 29 & 42,6 \\
Lesão Pulmonar na sepse & & \\
$\quad$ Lesão pulmonar aguda & 40 & 58,82 \\
$\quad$ SDRA & 18 & 26,5 \\
$\quad$ Outras & 10 & 14,8 \\
$\quad$ Óbito & 2 & 2,66 \\
Tempo médio de permanência (dias) & $10,9 \pm 7,1$ & \\
média \pm DP & & \\
Mediana (amplitude) & $9(2-39)$ & \\
Ângulo de fase ( ${ }^{\circ}$ ), média \pm DP & $3,24 \pm 1,15$ & \\
\hline
\end{tabular}

SDRA = síndrome do desconforto respiratório agudo

Tabela 2 - Média de Tempo de Internação dos Pacientes Sépticos

\begin{tabular}{lcc}
\hline & $\mathrm{N}$ & Média $\pm \mathrm{DP}$ \\
\hline Choque séptico & 27 & $14,5 \pm 8,8$ \\
Sepse grave & 29 & $10,9 \pm 6,2$ \\
Sepse & 12 & $7,3 \pm 3,4$ \\
\hline
\end{tabular}

Valor de $p=0,003$, associado ao teste Kruskal-Wallis. 
$\mathrm{Na}$ avaliação do escore PRISM I demonstrou-se que $70,7 \%$ dos pacientes (53/75) apresentaram escore de baixa e moderada probabilidade de morte (PRISM I $\leq$ $15 \%)$ nas primeiras $24 \mathrm{~h}$ de admissão, com ocorrência de um óbito, e 20,6\% (14/75) apresentaram alta e muito alta probabilidade de morte (PRISM I $>15 \%$ ), ocorrendo um óbito neste grupo. Dentre os óbitos (2/73) um foi na faixa etária de 1 ano de vida com choque séptico e o outro na faixa etária de $>72,1-120$ meses com sepse grave.

Neste estudo não se demonstrou relação estatisticamente significativa entre as categorias de PRISM I e a gravidade da sepse (evolução para sepse, sepse grave e choque séptico) nos 68 pacientes com sepse ( $p=$ 0,58 , valor associado ao teste do Qui-quadrado). $\mathrm{Na}$ Análise de Variância (ANOVA) também não houve diferença entre os valores médios de PRISM I e a gravidade da sepse $(p=0,26)$.

\section{Análise da Bioimpedância - Avaliação do Ângulo de Fase}

A BIA foi realizada nas primeiras 48 horas em $58,7 \%$ dos pacientes (44/75), média de 3 dias $(\mathrm{DP} \pm 3,28)$ e mediana de 2 dias.

A distribuição das médias do ângulo de fase por faixa etária está apresentada na tabela 3.

Tabela 3 - Distribuição da Média de Ângulo de Fase por Faixa Etária

\begin{tabular}{lcc}
\hline Faixa etária & $\begin{array}{c}\text { Ângulo de Fase } \\
\left({ }^{\circ}\right) \text { Média } \pm \text { DP }\end{array}$ & $\mathrm{N}$ \\
\hline$<=1$ mês & $2,91 \pm 1,01$ & 6 \\
$>1$ mês -1 ano & $3,28 \pm 1,28$ & 29 \\
$>1$ ano -2 anos & $2,67 \pm 0,68$ & 9 \\
$>2$ anos -6 anos & $2,92 \pm 0,99$ & 14 \\
$>6$ anos -10 anos & $3,49 \pm 1,16$ & 9 \\
$>10$ anos & $4,22 \pm 0,91$ & 8 \\
\hline
\end{tabular}

$p=0,06$, associado à ANOVA.

Não houve diferença entre as médias dos ângulos de fase em relação às categorias de PRISM I e de sepse (Tabela 4). Entretanto, observou-se relação inversa entre os ângulos de fase e as categorias de PRISM I, estando os menores valores de ângulo de fase associados à maior probabilidade de óbito (Figura 1). Ressalta-se nessa figura que $50 \%$ dos pacientes com maior escore de PRISM (> $30 \%$ ) apresentaram os valores mais baixos de ângulo de fase, variando de $1,5^{\circ}$ a $2,2^{\circ}$.
Tabela 4 - Distribuição das Médias do Ângulo de Fase $\left(^{\circ}\right)$ de Acordo com as Categorias do Escore PRISM I e da Gravidade da Sepse

\begin{tabular}{lcc}
\hline Categorias PRISM I & Média $\pm \mathrm{DP}$ & $\mathrm{N}$ \\
\hline$<=1$ & $3,72 \pm 1,53$ & 8 \\
$1,1-5$ & $3,28 \pm 1,16$ & 35 \\
$5,1-15$ & $3,24 \pm 0,94$ & 18 \\
$15,1-30$ & $3,15 \pm 1,08$ & 9 \\
$>30,1$ & $2,31 \pm 1,11$ & 5 \\
Total & & 75 \\
$\mathrm{p}=0,32$ (ANOVA) & & \\
Categorias de sepse & & \\
$\quad$ Choque séptico & $3,2 \pm 1,0$ & 27 \\
$\quad$ Sepse grave & $3,4 \pm 1,2$ & 29 \\
$\quad$ Sepse & $3,3 \pm 1,4$ & 12 \\
$\quad$ Total & & 68 \\
$\mathrm{p}=0,74$ (ANOVA) & & \\
\hline
\end{tabular}

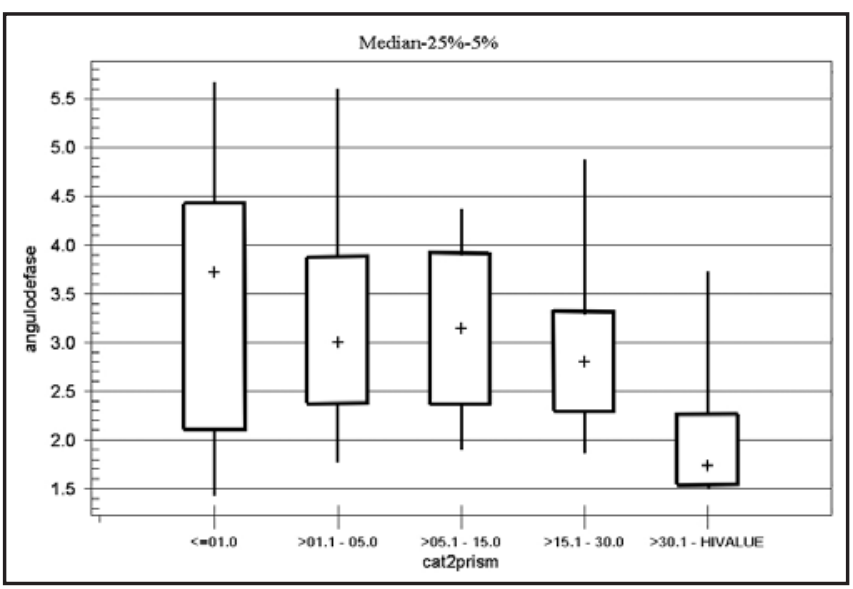

Figura 1 - Boxplot dos Valores de Ângulo de Fase em Relação aos Intervalos de Probabilidade de Mortalidade (PRISM I).

Ao comparar os ângulos de fase dos pacientes sépticos com DMOS observou-se relação inversa a partir do comprometimento de três órgãos, ou seja, quanto maior o comprometimento de órgãos menor foi o valor do ângulo de fase (Figura 2). Destaca-se nessa figura que pacientes que tiveram até dois órgãos comprometidos, apresentaram variabilidade maior do ângulo de fase, enquanto pacientes com comprometimento de três ou mais órgãos apresentaram variabilidade menor, com os ângulos de fase se distribuindo mais homogeneamente e com valores mais baixos. 


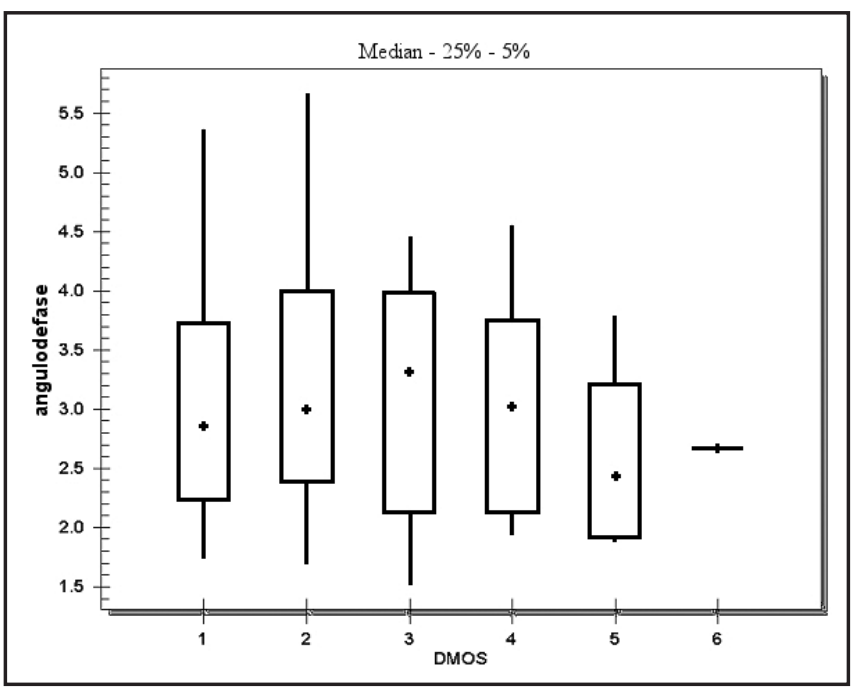

Figura 2 - Boxplot dos Valores de Ângulo de Fase de acordo com o Número de Órgãos Comprometidos na Disfunção de Múltiplos Órgãos e Sistemas (DMOS).

Para avaliar os valores de ângulo de fase com tempo de permanência dos casos de sepse foi necessário excluir três pacientes que apresentaram valores atípicos (17, 34 e 39 dias). Tais valores ocorreram porque eram pacientes crônicos, cuja permanência prolongada justificou-se pela doença de base e não pela evolução da sepse. A regressão linear mostrou uma tendência de que quanto maior o ângulo de fase menor foi o tempo de internação. Esta tendência manteve-se na análise das categorias de sepse grave e choque séptico. Entretanto, nos pacientes com choque séptico esta tendência foi mais evidente, apesar de não alcançar estatística significativa ( $R 2=-0,10 ; R=-0,32 ; p=0,12$ ). Neste estudo não foi possível demonstrar diferença significativa entre os valores de ângulo de fase e a lesão pulmonar aguda na sepse e síndrome do desconforto respiratório agudo, ou com a evolução para alta ou óbito (respectivamente $p=0,75$ e $p=0,50$ pelo teste $t$ de Student).

\section{DISCUSSÃo}

O ângulo de fase tem sido investigado na última década como um instrumento de valor prognóstico, nutricional, de função de membrana celular ou indicador de saúde em várias condições clínicas.

Apesar de não se dispor de uma distribuição dos valores do ângulo de fase em crianças brasileiras saudáveis para comparar os resultados encontrados, observou-se que em relação ao estudo de De Palo e col. em crianças saudáveis italianas, os valores do presente estudo foram mais baixos ${ }^{22}$. Eles observaram em 2044 crianças saudáveis a variação da média do AF de $4,6^{\circ}$ - 5,7 $7^{\circ}$ na faixa etária de 2 anos a 9 anos, e entre $5,7^{\circ}$ e $6,2^{\circ}$ de 10 a 15 anos. Outros estudos em adultos referem o valor de $3^{\circ}$ a $15^{\circ}$. O estudo de Nagano e col. ${ }^{13}$, realizado em pacientes desnutridos pediátricos, com idade entre 2 meses e 7 anos, também revelou valores baixos de ângulo de fase $\left(0,7^{\circ}\right.$ a $3,1^{\circ}$ - valores individuais), semelhante ao atual estudo.

Observou-se clara tendência dos pacientes com escores de PRISM I mais elevados apresentarem os menores valores de ângulo de fase. Entretanto, o PRISM I não foi útil em prever prognóstico em relação à gravidade da sepse quando relacionado com as apresentações mais graves de choque séptico e sepse grave. A principal crítica aos escores prognósticos é que os indicadores construídos a partir de dados obtidos na admissão ou nas primeiras 24 horas apenas descrevem o prognóstico dos pacientes críticos por categoria de risco. O ângulo de fase talvez possa ser um indicador evolutivo no acompanhamento destes pacientes. $\mathrm{O}$ escore de PRISM III que tem maior capacidade preditiva não foi avaliado neste estudo, em razão de seu elevado custo, o que tem limitado seu uso mesmo em países desenvolvidos ${ }^{23}$.

Mattar e col. compararam pacientes adultos sépticos e não sépticos pela bioimpedância destacando a utilização do ângulo de fase como indicador prognóstico ${ }^{8}$. Por definição, o ângulo de fase é positivamente associado com reactância e negativamente associado com resistência, e sua variação indica alterações, ou na composição corporal ou na função da membrana celular. Os pacientes com SIRS, sepse, sepse grave e choque séptico apresentaram profundas alterações entre o conteúdo de massa magra, água corporal total e na sua distribuição. $O$ aumento da permeabilidade capilar e a ruptura do metabolismo de membrana comumente vistos neste pacientes são responsáveis pelo extravasamento de líquidos, desenvolvimento do $3^{\circ}$ espaço e hidratação da porção lipofílica da membrana celular, o que explica os valores de Xc diminuídos e as alterações de $R$ encontradas neste estudo ${ }^{8}$. Embora a $R$ tenha variado com o grau de hidratação, a diminuição da $X_{c}$ foi proporcionalmente maior do que a diminuição da $R$, que resultou em diminuição do ângulo de fase. Os pacientes sépticos que não sobreviveram apresentaram AF na fase final da evolução de $3,9^{\circ} \pm 1,2^{\circ}$, os sépticos sobreviventes $5,2^{\circ} \pm 1,5^{\circ}$, comparados com $6,7^{\circ} \pm 1,7^{\circ}$ nos pacientes não sépticos ${ }^{8}$. Os valores dos pacientes 
sépticos não sobreviventes de estudo de Mattar e col. são semelhantes aos obtidos dos pacientes sépticos do presente estudo. Não há dados disponíveis publicados em pacientes sépticos pediátricos críticos que permitam comparações.

Os pacientes críticos apresentaram desidratação celular progressiva e proteólise. Finn e col. demonstraram que esses pacientes perdem $15 \%$ a $20 \%$ do seu conteúdo de potássio e proteína em cerca de 21 dias de doença e o volume celular também diminui cerca de $15 \%$ a $20 \%{ }^{24}$. O controle do volume celular é regulado por hormônios, substratos fisiológicos como aminoácidos e estresse oxidativo ${ }^{24}$. Estudos demonstraram que a diminuição da água intracelular (AIC) e o aumento da extracelular (AEC) são indicadores da reação catabólica da sepse, se não participantes da sua patogênese. A estimativa da AIC pela BIA é menos disponível do que a estimativa da AEC e da $\mathrm{ACT}^{25,26}$. Entretanto, estas estimativas dependem de equações não padronizadas para estes pacientes, especialmente crianças menores de 10 anos. O ângulo de fase por sua vez é um indicativo deste equilíbrio celular e mostrou-se no presente estudo alterado nas situações de maior gravidade, como na sepse grave e no choque séptico.

Estes efeitos no metabolismo da membrana celular são mediados por citocinas inflamatórias, especialmente o fator de necrose tumoral. A relação do polimorfismo destes mediadores, que hoje se sabe está associada com a morbidade, mortalidade e prognóstico, provavelmente também mostrará associação com a bioimpedância através do ângulo de fase (dados em fase de análise). São estes mediadores também os responsáveis pelo desenvolvimento da SDRA e da DMOS. Apesar do estudo não ter conseguido demonstrar relação entre o ângulo de fase e evolução para SDRA, observou-se uma relação entre baixos valores de ângulo de fase e maior número de órgãos comprometidos na DMOS. Esta tendência de valores mais baixos do ângulo de fase foi também evidente no tempo de internação, principalmente dos pacientes mais graves, que foram os de choque séptico.

A associação do ângulo de fase com mortalidade, já documentada em pacientes com sepse ${ }^{8}$, síndrome da imunodeficiência adquirida (HIV $)^{14}$, grandes queima$\operatorname{dos}^{27}$, câncer ${ }^{28}$, não foi demonstrada no grupo estudado, provavelmente em razão da mortalidade baixa ( 2 óbitos - 2,66\%).

O ângulo de fase como indicador nutricional nos pacientes críticos talvez possa ser usado em combinação com métodos antropométricos, criando-se um novo escore de avaliação, à semelhança do que foi proposto em pacientes com insuficiência renal submetido a procedimentos dialíticos ${ }^{29}$. O AF avalia diferentes dimensões do estado nutricional, o que implica em maior poder prognóstico. Este aspecto não foi investigado no presente estudo.

Uma limitação deste estudo deve-se ao fato do PRISM I que por definição é realizado nas primeiras 24 horas da admissão, ter sido comparado à BIA, que em cerca de $42 \%$ dos casos foi realizada após 48 horas. Atenção maior deve ser dada ao momento da realização da BIA para que possam ser corrigidos possíveis vieses de interpretação.

Diante do exposto, a partir desta análise inicial, encontrou-se uma tendência de associação entre ângulo de fase, PRISM I, evolução para DMOS e tempo de internação. Acredita-se que estudo que está sendo desenvolvido com número maior de pacientes, incluindo avaliação nutricional, possam demonstrar resultados mais robustos em relação às evidências destas associações. A caracterização dos valores de ângulo de fase na população de crianças brasileiras saudáveis é importante para que os dados possam ser comparados. Assim também, será interessante comparar os valores de AF, $\mathrm{R}$ e Xc no momento pré-alta dos pacientes graves.

\section{AGRADECIMENTOS}

Agradecemos ao Instituto Fernandes Figueira; a Dra. Glória Valéria da Veiga da Universidade Federal do Rio de Janeiro por ter cedido seu equipamento para coleta de dados; ao Sr. Rubens Rodrigues Barrozo e a Sra. Simone Cardoso de Mendonça Sales por todo empenho na aquisição dos suprimentos necessários à pesquisa; a equipe médica e de enfermagem da UPG pela colaboração na coleta dos dados. Este trabalho foi financiado pelo Programa de Desenvolvimento Tecnológico em Saúde Pública (PDTSP- FIOCRUZ).

\section{REFERÊNCIAS}

01. Slater A, Shann F, Pearson G - PIM2: a revised version of the Paediatric Index of Mortality. Intensive Care Med, 2003;29:278-285.

02. Seneff M, Knaus WA - Predicting patient outcome from intensive care: a guide to APACHE, MPM, SAPS, PRISM, and other prognostic scoring systems. J Intensive Care Med, 1990;5:33-52.

03. Pollack MM, Patel KM, Ruttimann UE - PRISM III: an update Pediatric Risk of Mortality score. Crit Care Med, 1996;24:743-752.

04. Gunning K, Rowan K - ABC of intensive care: outcome data and score systems. BMJ, 1999;319:241-244.

05. Lacroix J, Cotting $\mathrm{J}$ - Severity of illness and organ dysfunction scoring in children. Pediatr Crit Care Med, 2005;6:(Suppl3):S126-S134.

06. Rosenfeld RS - Avaliação nutricional: o que há de novo? Rev Bras Clin, 1998;13:101-107. 
07. Aloia JF, Vaswani A, Flaster E et al - Relationship of body water compartments to age, race, and fat-free mass. J Lab Clin Med, 1998;132:483-490.

08. GIBI Brazilian Group for Bioimpedance Study. Total body bioelectrical impedance measurement as a progressive outcome prediction and therapeutic index in the comparison between septic and non septic patients. A multicenter Brazilian study. Rev Metab Nutr, 1995;2:159-170.

09. Kotler DP, Burastero S, Wang J et al - Prediction of body cell mass, fatfree mass, and total body water with bioelectrical impedance analysis: effects of race, sex, and disease. Am J Clin Nutr, 1996;64:(Suppl3):489S497S.

10. Coppini LZ, Bottoni A, Teixeira Silva $M L$ et al - Aplicação da análise da impedância bioelétrica na avaliação nutricional. Rev Bras Clin, 1998;13:81-89.

11. Barbosa-Silva MC, Barros AJ, Post CL et al - Can bioelectrical impedance analysis identify malnutrition in preoperative nutritional assessment? Nutrition, 2003;19:422-426.

12. Barbosa-Silva MC, Barros AJ - Bioelectrical impedance analysis in clinical practice: a new perspective on its use beyond body composition equations. Curr Opin Clin Nutr Metab Care. 2005;8:311-317.

13. Nagano M, Suita S, Yamanouchi T - The validity of bioelectrical impedance phase angle for nutritional assessment in children. J Pediatr Surg, 2000;35:1035-1039.

14. Schwenk A, Beisenherz A, Romer $\mathrm{K}$ et al - Phase angle from bioelectrical impedance analysis remains an independent predictive marker in HIVinfected patients in the era of highly active antiretroviral treatment. Am J Clin Nutr, 2000;72:496-501.

15. Barbosa-Silva MC, Barros AJ - Bioelectrical impedance and individual characteristics as prognostic factors for post-operative complications. Clin Nutr, 2005;24:830-838.

16. Scheltinga MR, Kimbrough TD, Jacobs DO et al - Altered cell membrane function in critical illness can be characterized by measuring body reactance. Surg Forum, 1990;41:43-44.

17. Goldstein B, Giroir B, Randolph A - International pediatric sepsis consensus conference: definitions for sepsis and organ dysfunction in pe- diatrics. Pediatr Crit Care Med, 2005;6:2-8.

18. Bernard GR, Artigas A, Brigham KL et al - The American-European Consensus Conference on ARDS. Definitions, mechanisms, relevant outcomes, and clinical trial coordination. Am J Respir Crit Care Med, 1994;149:818-824.

19. Murray JF, Matthay MA, Luce JM et al - An expanded definition of adult respiratory distress syndrome. Am Rev Respir Dis, 1988;138:720-723.

20. Gemke RJ, van Vught $\mathrm{J}$ - Scoring systems in pediatric intensive care: PRISM III versus PIM. Intensive Care Med, 2002;28:204-207.

21. Baumgartner RN, Chumlea WC, Roche AF - Bioelectric impedance phase angle and body composition. Am J Clin Nutr, 1988;48:16-23.

22. De Palo T, Messina G, Edefonti $A$ et al - Normal values of the bioelectrical impedance vector in childhood and puberty. Nutrition, 2000;16:417424.

23. Martha VF, Garcia PC, Piva JP et al - Comparison of two prognostic scores (PRISM and PIM) at a pediatric intensive care unit. J Pediatr, 2005;81:259-264.

24. Finn PJ, Plank LD, Clark MA et al - Progressive cellular dehydration and proteolysis in critically ill patients. Lancet, 1996;347:654-656.

25. Schwenk A, Schlottmann S, Kremer G et al - Fever and sepsis during neutropenia are associated with expansion of extracellular and loss of intracellular water. Clin Nutr, 2000;19:35-41.

26. Frankenfield DC, Cooney RN, Smith JS et al - Bioelectrical impedance plethysmographic analysis of body composition in critically injured and healthy subjects. Am J Clin Nutr, 1999;69:426-431.

27. Zdolsek HJ, Lindahl OA, Angquist KA et al - Non-invasive assessment of intercompartmental fluids shifts in burn victims. Burns. 1998;24:233240.

28. Toso S, Piccoli A, Gusella M et al - Altered tissue electrical properties lung cancer patients as detected by bioelectrical impedance vector analysis. Nutrition, 2000;16:120-124.

29. Edefonti A, Picca M, Damiani B et al - Prevalence of malnutrition assessed by bioimpedance analysis and anthropometry in children on peritoneal dialysis. Perit Dial Int, 2001;21:172-179. 